\title{
THE RESOLUTION OF ANY COLLINEATION INTO
}

\section{PERSPECTIVE REFLECTIONS*}

BY

M. W. HASKELL

The general formulæ of a collineation being

$$
\rho y_{i}=\sum_{k=1}^{k=n} a_{i k} x_{k},
$$

I shall consider only collineations which have $n$ distinct fixed points and can therefore be reduced to the normal form

$$
\rho y_{i}=m_{i} x_{i} \text {. }
$$

These multipliers $m_{i}$ are the roots of the characteristic equation of the collineation

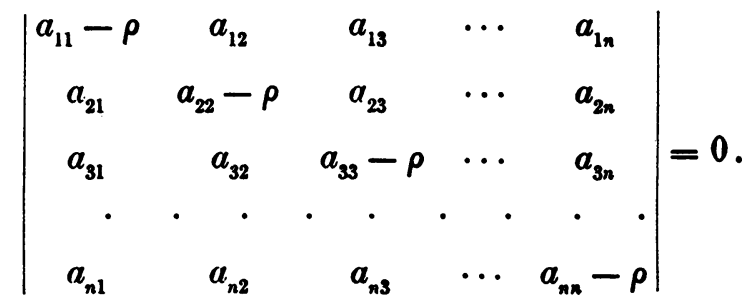

Just as any substitution can be resolved into a product of simple transpositions, so it will be shown that any collineation can be resolved into a product of perspective reflections, i. e., involutory collineations, and the minimum number of such perspective reflections will be determined. For the sake of clearness, the cases of the line, plane and ordinary space will be treated separately; the result for space of $n$ dimensions is then deduced easily.

By definition a perspective reflection is one which leaves invariant a point $\kappa\left(\kappa_{1}: \kappa_{2}: \cdots: \kappa_{n+1}\right)$ and every point in a flat space of $n-1$ dimensions, not containing $\kappa$ and denoted by $a\left(a_{1}: a_{2}: \ldots: a_{n+1}\right)$ or by the equation

* Presented to the Sooiety at the Congress of Arts and Science at St. Lonis, September 22, 1904. Received for publication January 11, 1905. 
$a_{x} \equiv a_{1} x_{1}+a_{2} x_{2}+\cdots+a_{n+1} x_{n+1}=0$, and which converts any point into its harmonic conjugate with respect to the point $\kappa$ and the space $a$.

Hence the general formulæ for such a collineation may be written

$$
\rho y_{i}=a_{\kappa} x_{i}-2 \kappa_{i} a_{x},
$$

the determinant of which is equal to $-a_{\kappa}^{n+1}$. By such a collineation every quadric space of $n-1$ dimensions with respect to which $\kappa$ and $a$ are pole and polar is obviously converted into itself. A perspective reflection will ordinarily be denoted by $T$, or by $T(a, \kappa)$ if it is desirable to put the elements $a$ and $\kappa$ in evidence.

\section{Linear transformations of a single variable.}

We can hardly use the word collineation in this case, but the general formulæ can be used, and will be used for the sake of uniformity.

THEOREM. The general linear transformation of a single variable can be resolved into the product of two perspective reflections of period two, and in $\infty^{\prime}$ ways.

If the general linear transformation $S$ be reduced to its normal form

$$
S: \quad \rho y_{i}=m_{i} x_{i}
$$

and be multiplied by the transformation $T(a, \kappa)$ of period two

$$
T: \quad \rho y_{i}=a_{\kappa} x_{i}-2 \kappa_{i} a_{x},
$$

the product $S T=U$ will be

$$
\begin{aligned}
U: \quad & \rho y_{1}=\left(-a_{1} \kappa_{1}+a_{2} \kappa_{2}\right) m_{1} x_{1}-2 a_{2} \kappa_{1} m_{2} x_{2}, \\
& \rho y_{2}=-2 a_{1} \kappa_{2} m_{1} x_{1}+\left(a_{1} \kappa_{1}-a_{2} \kappa_{2}\right) m_{2} x_{2} .
\end{aligned}
$$

This transformation $U$ will itself be of period two if the roots of its characteristic equation

$$
\rho^{2}-\rho\left(a_{1} \kappa_{1}-a_{2} \kappa_{2}\right)\left(m_{2}-m_{1}\right)-a_{\kappa}^{2} m_{1} m_{2}=0
$$

are equal and opposite, that is to say, if $\kappa_{1}: \kappa_{2}=a_{2}: a_{1}$. Then $T$ reduces to

and $U$ becomes

$$
y_{1}: y_{2}=a_{2}^{2} x_{2}: a_{1}^{2} x_{1}
$$

$$
y_{1}: y_{2}=a_{2}^{2} m_{2} x_{2}: a_{1}^{2} m_{1} x_{1} \text {. }
$$

It is evident that $U$ is of period two, and since $S T=U$, it follows that $S=U T$, which was to be proved. Moreover since $a_{1}: a_{2}$ is arbitrary, excluding of course the two cases $a_{1}: a_{2}=1: 0$ and $a_{1}: a_{2}=0: 1$, the resolution can be effected in $\infty^{1}$ ways. 


\section{Collineations in the plane.}

The product of two perspective reflections $T_{1}(a, \kappa)$ and $T_{2}(b, \lambda)$ will leave invariant the point $\mu$ where $a$ and $b$ meet and the two points on the line $(\kappa \lambda)$ which are harmonically conjugate with respect to both $(a, \kappa)$ and $(b, \lambda)$. If we take the triangle $(\kappa \lambda \mu)$ as coördinate triangle, the various elements involved will be

$$
\begin{array}{ll}
\kappa(1: 0: 0), & \lambda(0: 1: 0), \\
a\left(a_{1}: a_{2}: 0\right), & b\left(b_{1}: b_{2}: 0\right),
\end{array}
$$

and the formulæ of the collineations $T_{1}$ and $T_{2}$ become

$$
\begin{aligned}
& T_{1}: \quad \rho y_{1}=-a_{1} x_{1}-2 a_{2} x_{2}, \quad T_{2}: \quad \rho y_{1}=b_{2} x_{1}, \\
& \rho y_{2}=a_{1} x_{2}, \quad \rho y_{2}=-2 b_{1} x_{1}-b_{2} x_{2} \text {, } \\
& \rho y_{3}=a_{1} x_{3}, \quad \rho y_{3}=b_{2} x_{3} .
\end{aligned}
$$

From this we deduce

$$
\begin{array}{ll}
T_{1} T_{2}: & \rho y_{1}=-a_{1} b_{2} x_{1}-2 a_{2} b_{2} x_{2}, \\
& \rho y_{2}=2 a_{1} b_{1} x_{1}+\left(4 a_{2} b_{1}-a_{1} b_{2}\right) x_{2}, \\
& \rho y_{2}=a_{1} b_{2} x_{3},
\end{array}
$$

of which the characteristic equation is

$$
\left(a_{1} b_{2}-\rho\right)\left[\rho^{2}-\rho\left(4 a_{2} b_{1}-a_{1} b_{2}\right)+\left(a_{1} b_{2}\right)^{2}\right]=0 .
$$

In this equation the product of two of the roots is equal to the square of the third, $\rho_{1} \rho_{2}=\rho_{3}^{2}$, and the collineation $T_{1} T_{2}$ is therefore reducible to the normal form

$$
S: \quad \rho y_{1}=m_{1} x_{1}, \quad \rho y_{2}=m_{2} x_{2}, \quad \rho y_{3}=x_{3},
$$

where $m_{1} m_{2}=1$. This collineation converts into itself every conic $x_{1} x_{2}+k x_{3}^{2}=0$, the point $(0: 0: 1)$ being the polar of the line $x_{3}=0$. Conversely :

Every plane collineation which leaves a conic invariant can be resolved into the product of two perspective reflections, and this may be effected in $\infty^{1}$ ways.

For it can be shown that every such collineation $S$ can be reduced to the normal form $S$ just given. With $S$ we must compound a reflection $T_{2}(a, \kappa)$, where $x$ lies on $x_{3}=0$ and $a$ passes through $(0: 0: 1)$, that is,

$$
\kappa=\kappa\left(\kappa_{1}: \kappa_{2}: 0\right), \quad a=a\left(a_{1}: a_{2}: 0\right), \quad a_{\kappa}=a_{1} \kappa_{1}+a_{2} \kappa_{2} .
$$

The formulæ of $T_{2}$ are therefore

$$
\begin{array}{ll}
T_{2}: \quad \rho y_{1}=\left(-a_{1} \kappa_{1}+a_{2} \kappa_{2}\right) x_{1}-2 a_{2} \kappa_{1} x_{2}, \\
\\
\rho y_{2}=-2 a_{1} \kappa_{2} x_{1}+\left(a_{1} \kappa_{1}-a_{2} \kappa_{2}\right) x_{2}, \\
\rho y_{3}=\left(a_{1} \kappa_{1}+a_{2} \kappa_{2}\right) x_{3} .
\end{array}
$$


and the characteristic equation of $S T_{2}$ is

$$
\left(a_{\kappa}-\rho\right)\left[\rho^{2}-\rho\left(a_{1} \kappa_{1}-a_{2} \kappa_{2}\right)\left(m_{2}-m_{1}\right)-a_{\kappa}^{2}\right]=0 .
$$

Hence $S T_{2}=T_{1}$ will itself be a perspective reflection if $a_{1} \kappa_{1}-a_{2} \kappa_{2}=0$, that is, if $a_{1}: a_{2}=1 / \kappa_{1}: 1 / \kappa_{2}$, where $\kappa_{1}: \kappa_{2}$ is arbitrary, only the points $1: 0: 0$ and $0: 1: 0$ being excluded. From $S T_{2}=T_{1}$ and $T_{2}^{2}=1$, follows $S=T_{1} T_{2}$, and it is clear that this resolution may be effected in $\infty^{1}$ ways.

It will now be shown that corresponding to any plane collineation $U$, there are $\infty^{3}$ perspective reflections $T_{3}$ such that the product $U T_{3}=S$ will be of the type just considered, leaving a conic unchanged. If $U$ be written in the normal form

$$
U: \quad \rho y_{i}=m_{i} x_{i}
$$

and $T_{3}$ in the general form

$$
T_{3}: \quad \rho y_{i}=a_{\kappa} x_{i}-2 \kappa_{i} a_{x},
$$

the formulæ for $U T_{3}$ become

$$
\begin{aligned}
& \rho y_{1}=\left(a_{\kappa}-2 a_{1} \kappa_{1}\right) m_{1} x_{1}-2 a_{2} \kappa_{1} m_{2} x_{2}-2 a_{3} \kappa_{1} m_{3} x_{3}, \\
& \rho y_{2}=-2 a_{1} \kappa_{2} m_{1} x_{1}+\left(a_{\kappa}-2 a_{2} \kappa_{2}\right) m_{2} x_{2}-2 a_{3} \kappa_{2} m_{3} x_{3}, \\
& \rho y_{3}=-2 a_{1} \kappa_{3} m_{1} x_{1}-2 a_{2} \kappa_{3} m_{2} x_{2}+\left(a_{\kappa}-2 a_{3} \kappa_{3}\right) m_{3} x_{3} .
\end{aligned}
$$

The characteristic equation of this collineation is

$$
\begin{aligned}
-\rho^{3}+\rho^{2}\left[\left(a_{\kappa}-2 a_{1} \kappa_{1}\right) m_{1}\right. & \left.+\left(a_{\kappa}-2 a_{2} \kappa_{2}\right) m_{2}+\left(a_{\kappa}-2 a_{2} \kappa_{3}\right) m_{3}\right] \\
-\rho a_{\kappa}\left[m_{2} m_{3}\left(a_{\kappa}-2 a_{2} \kappa_{2}-2 a_{3} \kappa_{3}\right)+m_{3} m_{1}\left(a_{\kappa}-2 a_{3} \kappa_{3}-2 a_{1} \kappa_{1}\right)\right. & \\
& \left.+m_{1} m_{2}\left(a_{\kappa}-2 a_{1} \kappa_{1}-2 a_{2} \kappa_{2}\right)\right]-a_{\kappa}^{3} m_{1} m_{2} m_{3}=0 .
\end{aligned}
$$

If now $U T_{3}$ is to leave a conic unchanged, the roots of this characteristic equation must satisfy the relation $\rho_{1} \rho_{2}=\rho_{3}^{2}$, whence

$$
\left(\rho_{1} \rho_{2}+\rho_{2} \rho_{3}+\rho_{3} \rho_{1}\right)^{3}=\rho_{1} \rho_{2} \rho_{3}\left(\rho_{1}+\rho_{2}+\rho_{3}\right)^{3},
$$

or the coefficients of the equation satisfy the relation

$$
\left[\sum m_{2} m_{3}\left(a_{\kappa}-2 a_{2} \kappa_{2}-2 a_{3} \kappa_{3}\right)\right]^{3}+m_{1} m_{2} m_{3}\left[\sum m_{1}\left(a_{\kappa}-2 a_{1} \kappa_{1}\right)\right]^{3}=0 .
$$

Here $\kappa$ may be chosen arbitrarily, excluding only the fixed points of $U$, and then this condition becomes the equation of three points, through one of which $a$ must pass. Then $T_{3}$ may be found in $\infty^{2} \cdot \infty^{1}=\infty^{3}$ ways so that $U T_{3}=S$ shall be of the required type.

Combining these two results, we have the following

Theorem. Any plane collineation $U$ can be resolved in $\infty^{4}$ ways into the product of three perspective reflections $T_{1} T_{2} T_{3}$. 
A case of especial interest and importance in that in which

$$
\sum m_{1}\left(a_{\kappa}-2 a_{1} \kappa_{1}\right)=0, \quad \sum m_{2} m_{3}\left(a_{\kappa}-2 a_{2} \kappa_{2}-2 a_{3} \kappa_{3}\right)=0,
$$

for in this case $S^{3}=1$. The solution of these equations gives $a_{1} \kappa_{1}: a_{2} \kappa_{2}: a_{3} \kappa_{3}$

$$
=\left(m_{2}-m_{3}\right)\left(m_{2} m_{3}+m_{1}^{2}\right):\left(m_{3}-m_{1}\right)\left(m_{3} m_{1}+m_{2}^{2}\right):\left(m_{1}-m_{2}\right)\left(m_{1} m_{2}+m_{3}^{2}\right) .
$$

A number of well-known groups are generated by collineations $S$ and 1 , where

$$
S^{n}=1, \quad T^{2}=1, \quad(S T)^{3}=1,
$$

and the analysis just given readily furnishes the complete formulæ.

\section{Collineations in space of three dimensions.}

The product of two reflections in space $T_{1}(a, \kappa)$ and $T_{2}(b, \lambda)$ will leave invariant every point on the line of intersection of the planes $a$ and $b$, together with two points on the line $(\kappa \lambda)$. It can be shown just as above that the normal form of the product $T_{1} T_{2}$ will be

where $m_{1} m_{2}=1$.

$$
y_{1}: y_{2}: y_{3}: y_{4}=m_{1} x_{1}: m_{2} x_{2}: x_{3}: x_{4},
$$

The product of three perspective reflections $T_{1}(\alpha, \kappa), T_{2}(b, \lambda)$ and $T_{3}(c, \mu)$ will leave invariant the point $\nu$ common to $a, b$ and $c$, and also three points on the plane $(\kappa \lambda \mu)$. Taking $\kappa, \lambda, \mu, \nu$ as the vertices of the tetrahedron of reference, we have

$$
\begin{array}{lll}
\kappa=\kappa(1: 0: 0: 0), & \lambda=\lambda(0: 1: 0: 0), & \mu=\mu(0: 0: 1: 0), \\
a=a\left(a_{1}: a_{2}: a_{3}: 0\right), & b=b\left(b_{1}: b_{2}: b_{3}: 0\right), & c=c\left(c_{1}: c_{2}: c_{3}: 0\right),
\end{array}
$$

and the formulæ of $T_{1}$ are simply

$$
\begin{aligned}
T_{1}: \quad & \rho y_{1}=-a_{1} x_{1}-2 a_{2} x_{2}-2 a_{3} x_{3}, \\
& \rho y_{2}=a_{1} x_{2}, \\
& \rho y_{3}=a_{1} x_{3}, \\
& \rho y_{4}=a_{1} x_{4},
\end{aligned}
$$

with similar formulæ for $T_{2}$ and $T_{3}$. Hence we have for $T_{1} T_{2} T_{3}$ the formulæ

$$
\begin{aligned}
& \rho y_{1}=-a_{1} b_{2} c_{3} x_{1}-2 a_{2} b_{2} c_{3} x_{2}-2 a_{3} b_{2} c_{3} x_{3} \text {, } \\
& \rho y_{2}=2 a_{1} b_{1} c_{3} x_{1}+\left(4 a_{2} b_{1} c_{3}-a_{1} b_{2} c_{3}\right) x_{2}+\left(4 a_{3} b_{1} c_{3}-2 a_{1} b_{3} c_{3}\right) x_{3} \text {, } \\
& \rho y_{3}=\left(2 a_{1} b_{2} c_{1}-4 a_{1} b_{1} c_{2}\right) x_{1}+\left(4 a_{2} b_{2} c_{1}-8 a_{2} b_{1} c_{2}+2 a_{1} b_{2} c_{2}\right) x_{2} \\
& \rho y_{4}=a_{1} b_{2} c_{3} x_{4} \\
& +\left(4 a_{3} b_{2} c_{1}-8 a_{3} b_{1} c_{2}+4 a_{1} b_{3} c_{2}-a_{1} b_{2} c_{3}\right) x_{3} \text {, }
\end{aligned}
$$


and the characteristic equation is

$$
\begin{gathered}
\left(a_{1} b_{2} c_{3}-\rho\right)\left[-\rho^{3}+\rho^{2}\left(-8 a_{3} b_{1} c_{2}+4 a_{1} b_{3} c_{2}+4 a_{3} b_{2} c_{1}+4 a_{2} b_{1} c_{3}-3 a_{1} b_{2} c_{3}\right)\right. \\
\left.-\rho\left(a_{1} b_{2} c_{3}\right)\left(8 a_{2} b_{3} c_{1}-4 a_{1} b_{3} c_{2}-4 a_{3} b_{2} c_{1}-4 a_{2} b_{1} c_{3}+3 a_{1} b_{2} c_{3}\right)-\left(a_{1} b_{2} c_{3}\right)^{3}\right]=0 .
\end{gathered}
$$

This equation is characterized by the property that $\rho_{1} \rho_{2} \rho_{3}+\rho_{4}^{3}=0$, and the normal form of the collineation will be $y_{1}: y_{2}: y_{3}: y_{4}=m_{1} x_{1}: m_{2} x_{2}: m_{3} x_{3}:-x_{4}$, where $m_{1} m_{2} m_{3}=1$.

I propose now to show that any collineation $S_{4}$ in space of three dimensions can be reduced to a product $S_{3} T_{4}$ where the multipliers of $S_{3}$ are connected by the relation $m_{1} m_{2} m_{3}+m_{4}^{3}=0$ and $T_{4}$ is a reflection; that a collineation of type $S_{3}$ can be reduced to a product $S_{2} T_{3}$ where the multipliers of $S_{2}$ satisfy the relations $m_{1} m_{2}=m_{3}^{2}, m_{3}=m_{4}$, and $T_{3}$ is a reflection; finally, that a collineation of type $S_{2}$ can be reduced to a product of two reflections $T_{1} T_{2}$, and hence that $S_{4}=T_{1} T_{2} T_{3} T_{4}$.

If $S_{4}$ be reduced to the normal form

$$
\rho y_{i}=m_{i} x_{i}
$$

and $T_{4}$ be given in the general form

$$
\rho y_{i}=a_{\kappa} x_{i}-2 \kappa_{i} a_{x},
$$

then $S_{4} T_{4}=S_{3}$ will be of the form

$$
\rho y_{i}=a_{\kappa} m_{i} x_{i}-2 \kappa_{i} a_{m x},
$$

and the characteristic equation of $S_{3}$ will be

$$
\left|\begin{array}{cccc}
\left(a_{\kappa}-2 a_{1} \kappa_{1}\right) m_{1}-\rho & -2 a_{2} \kappa_{1} m_{2} & -2 a_{3} \kappa_{1} m_{3} & -2 a_{4} \kappa_{1} m_{4} \\
-2 a_{1} \kappa_{2} m_{1} & \left(a_{\kappa}-2 a_{2} \kappa_{2}\right) m_{2}-\rho & -2 a_{3} \kappa_{2} m_{3} & -2 a_{4} \kappa_{2} m_{4} \\
-2 a_{1} \kappa_{3} m_{1} & -2 a_{2} \kappa_{3} m_{2} & \left(a_{\kappa}-2 a_{3} \kappa_{3}\right) m_{3}-\rho & -2 a_{4} \kappa_{3} m_{4} \\
-2 a_{1} \kappa_{4} m_{1} & -2 a_{2} \kappa_{4} m_{2} & -2 a_{3} \kappa_{4} m_{3} & \left(a_{\kappa}-2 a_{4} \kappa_{4}\right) m_{4}-\rho
\end{array}\right|=0 .
$$

This reduces to

$$
\begin{aligned}
& \rho^{4}-\rho^{3} \sum\left(a_{\kappa}-2 a_{1} \kappa_{1}\right) m_{1}+\rho^{2} a_{\kappa} \sum m_{1} m_{2}\left(a_{\kappa}-2 a_{1} \kappa_{1}-2 a_{2} \kappa_{2}\right) \\
& -\rho a_{\kappa}^{2} \sum m_{1} m_{2} m_{3}\left(a_{\kappa}-2 a_{1} \kappa_{1}-2 a_{2} \kappa_{2}-2 a_{3} \kappa_{3}\right)-a_{\kappa}^{4} m_{1} m_{2} m_{3} m_{4}=0,
\end{aligned}
$$

and $S_{3}$ will be of the required character if this equation be satisfied by any fourth root of $a_{\kappa}^{4} m_{1} m_{2} m_{3} m_{4} ; T_{4}$ can therefore be chosen in $\infty^{5}$ different ways.

In particular, if the equations

$$
\begin{aligned}
\sum m_{1}\left(a_{\kappa}-2 a_{1} \kappa_{1}\right) & =0, \\
\sum m_{1} m_{2}\left(a_{\kappa}-2 a_{1} \kappa_{1}-2 a_{2} \kappa_{2}\right) & =0, \\
\sum m_{1} m_{2} m_{3}\left(a_{\kappa}-2 a_{1} \kappa_{1}-2 a_{2} \kappa_{2}-2 a_{3} \kappa_{3}\right) & =0
\end{aligned}
$$


be simultaneously satisfied, $S_{3}^{4}=1$; and in this case $T_{1}$ can be chosen in $\infty^{3}$ ways.

Reducing $S_{3}$ to its normal form

$$
y_{1}: y_{2}: y_{3}: y_{4}=m_{1} x_{1}: m_{2} x_{2}: m_{3} x_{3}:-x_{4},
$$

where $m_{1} m_{2} m_{3}=1$, we must choose the elements of $T_{3}(b, \lambda)$, so that $\lambda$ lies on $x_{4}=0$ and $b$ passes through $0: 0: 0: 1$, that is to say,

$$
\lambda=\lambda\left(\lambda_{1}: \lambda_{2}: \lambda_{3}: 0\right) \quad \text { and } \quad b=b\left(b_{1}: b_{2}: b_{3}: 0\right) \text {, }
$$

so that $b_{\lambda} \equiv b_{1} \lambda_{1}+b_{2} \lambda_{2}+b_{3} \lambda_{3}$. We can then write down the characteristic equation of $S_{3} T_{3}$ immediately as follows :

$$
\left|\begin{array}{cccc}
\left(b_{\lambda}-2 b_{1} \lambda_{1}\right) m_{1}-\rho & -2 b_{2} \lambda_{1} m_{2} & -2 b_{3} \lambda_{1} m_{3} & 0 \\
-2 b_{1} \lambda_{2} m_{1} & \left(b_{\lambda}-2 b_{2} \lambda_{2}\right) m_{2}-\rho & -2 b_{3} \lambda_{2} m_{3} & 0 \\
-2 b_{1} \lambda_{3} m_{1} & -2 b_{2} \lambda_{3} m_{2} & \left(b_{\lambda}-2 b_{2} \lambda_{2}\right) m_{3}-\rho & 0 \\
0 & 0 & 0 & -b_{\lambda}-\rho
\end{array}\right|=0 \text {. }
$$

This is equivalent to

$$
\begin{aligned}
-\left(b_{\lambda}+\rho\right)\left[-\rho^{3}+\rho^{2} \sum m_{1}\left(b_{\lambda}-2 b_{1} \lambda_{1}\right)\right. & \\
& \left.-\rho b_{\lambda} \sum m_{1} m_{2}\left(b_{\lambda}-2 b_{1} \lambda_{1}-2 b_{2} \lambda_{2}\right)-b_{\lambda}^{3}\right]=0 .
\end{aligned}
$$

One root of this equation is obviously $-b_{\lambda}$; and, that the product $S_{3} T_{3}=S_{2}$ be of the required form, it is necessary and sufficient that a second root should also be equal to $-b_{\lambda}$; hence

$$
\sum m_{1}\left(b_{\lambda}-2 b_{1} \lambda_{1}\right)+\sum m_{1} m_{2}\left(b_{\lambda}-2 b_{1} \lambda_{1}-2 b_{2} \lambda_{2}\right)=0 .
$$

This reduction can evidently be performed in $\propto^{3}$ ways, and $S_{2}$ can be reduced to the normal form

where $m_{1} m_{2}=1$.

$$
y_{1}: y_{2}: y_{3}: y_{4}=m_{1} x_{1}: m_{2} x_{2}: x_{3}: x_{4} \text {, }
$$

In particular, if

$$
\begin{aligned}
\sum m_{1}\left(b_{\lambda}-2 b_{1} \lambda_{1}\right) & =0, \\
\sum m_{1} m_{2}\left(b_{\lambda}-2 b_{1} \lambda_{1}-2 b_{2} \lambda_{2}\right) & =0,
\end{aligned}
$$

$S_{2}$ will be of period three; and this reduction can be effected in $\infty^{2}$ ways.

The elements of $T_{2}(c, \mu)$ must now be so chosen that $\mu$ lies on the line $x_{3}=0, x_{1}=0$, and $c$ passes through the line $x_{1}=0, x_{2}=0$. Then

$$
\mu=\mu\left(\mu_{1}: \mu_{2}: 0: 0\right) \text { and } c=c\left(c_{1}: c_{2}: 0: 0\right) \text {, }
$$


so that $c_{\mu}=c_{1} \mu_{1}+c_{2} \mu_{2}$. The characteristic equation of $S_{2} T_{2}=Z_{1}$ is then

$$
\begin{array}{cccc}
\left(c_{\mu}-2 c_{1} \mu_{1}\right) m_{1}-\rho & -2 c_{2} \mu_{1} m_{2} & 0 & 0 \\
-2 c_{1} \mu_{2} m_{1} & \left(c_{\mu}-2 c_{2} \mu_{2}\right) m_{2}-\rho & 0 & 0 \\
0 & 0 & c_{\mu}-\rho & 0 \\
0 & 0 & 0 & c_{\mu}-\rho
\end{array}=0
$$

and this is equivalent to

$$
\left(c_{\mu}-\rho\right)^{2}\left[\rho^{2}-\rho \Sigma m_{1}\left(c_{\mu}-2 c_{1} \mu_{1}\right)-c_{\mu}^{2}\right]=0 .
$$

$T_{1}$ will be a reflection if the coefficient of $\rho$ is equal to zero. This gives

$$
c_{1} \mu_{1}-c_{2} \mu_{2}=0 \text {, }
$$

and the reduction can be performed in $\infty^{1}$ ways.

We have then the final result:

THEOREM. Any collineation in space may be reduced to a product of four perspective reflections in $\infty^{9}$ ways. In particular, the reduction $S_{4}=T_{1} T_{2} T_{3} T_{4}$, subject to the relations $\left(T_{1} T_{2}\right)^{3}=1,\left(T_{1} T_{2} T_{3}\right)^{4}=1$ can be effected in $\infty^{6}$ ways.

\section{Collineations in space of $n$ dimensions.}

Passing now to the general case of collineations in space of $n$ dimensions we observe first that the product of $k+1$ reflections $(k \leqq n)$,

$$
T_{1}(a, \kappa), \quad T_{2}(b, \lambda), \quad \cdots \quad T_{k+1}(s, \sigma)
$$

leaves invariant all the points common to the spaces $a, b, \ldots, s$, together with $k+1$ points lying in the flat space of $k$ dimensions determined by the points $\kappa, \lambda, \cdots, \sigma$. The normal form of such a product is evidently

$$
\begin{array}{lr}
\rho y_{i}=m_{i} x_{i} & (i=1,2,3, \cdots, k+1), \\
\rho y_{i}=x_{i} & (i=k+2, k+3, \cdots, n+1),
\end{array}
$$

where $m_{1} m_{2} \cdots m_{k+1}=(-1)^{k+1}$.

Vice versa, any collineation $S_{k+1}$ which can be reduced to the above normal form can be resolved in $\infty^{k^{2}}$ ways into the product of $k+1$ perspective reflections, as I shall show immediately. We must evidently compound $S_{k+1}$ first with a perspective reflection $T_{k+1}(s, \sigma)$ for which

and hence

$$
\begin{aligned}
& s=s\left(s_{1}: s_{2}: \cdots s_{k+1}: 0: 0: \cdots 0\right), \\
& \sigma=\sigma\left(\sigma_{1}: \sigma_{2}: \cdots \sigma_{k+1}: 0: 0: \cdots 0\right),
\end{aligned}
$$

$$
s_{\sigma}=s_{1} \sigma_{1}+s_{2} \sigma_{2}+\cdots s_{k+1} \sigma_{k+1} \text {. }
$$


Then the characteristic equation of $S_{k+1} T_{k+1}$ will be

$$
\begin{aligned}
\left(s_{\sigma}-\rho\right)^{n-k}\left[(-\rho)^{k+1}+(-\rho)^{k} \sum m_{1}\left(s_{\sigma}-2 s_{1} \sigma_{1}\right)\right. & \\
+(-\rho)^{k-1} s_{\sigma} \sum m_{1} & m_{2}\left(s_{\sigma}-2 s_{1} \sigma_{1}-2 s_{2} \sigma_{0}\right) \\
& \left.+\cdots-m_{1} n_{2} \cdots m_{k+1} s_{\sigma}^{k+1}\right]=0 ;
\end{aligned}
$$

$(n-k)$ roots of this equation are obviously $=s_{\sigma}$; still another root will have this value if

$$
\begin{aligned}
& \sum m_{1}\left(s_{\sigma}-2 s_{1} \sigma_{1}\right)-\sum m_{1} m_{2}\left(s_{\sigma}-2 s_{1} \sigma_{1}-2 s_{2} \sigma_{2}\right)+\cdots \\
& +(-1)^{k-1} \sum m_{1} m_{2} \cdots m_{k}\left(s_{\sigma}-2 s_{1} \sigma_{1} \cdots 2 s_{k} \sigma_{k}\right)=0,
\end{aligned}
$$

where evidently $\sigma$ may be chosen arbitrarily, that is, in $\infty^{k}$ ways, and $s$ can then be chosen in $\infty^{k-1}$ ways. The normal form of $S_{k+1} T_{k+1}=S_{k}$ is then of the same form as that of $S_{k+1}, k$ being substituted for $k+1$, and the reduction can be performed in $\infty^{2 k-1}$ ways.

In particular, the collineation $S_{k}$ will be of period $k+1$ if all the terms in the above equation vanish simultaneously :

$$
\begin{aligned}
\sum m_{1}\left(s_{\sigma}-2 s_{1} \sigma_{1}\right) & =0, \\
\sum m_{1} m_{2}\left(s_{\sigma}-2 s_{1} \sigma_{1}-2 s_{2} \sigma_{2}\right) & =0, \\
\text { etc. } &
\end{aligned}
$$

In this case, there are exactly enough equations to determine the ratios

$$
s_{1} \sigma_{1}: s_{2} \sigma_{2}: \cdots: s_{k+1} \sigma_{k+1} \text {, }
$$

so that $\sigma$ may be chosen arbitrarily and $s$ is then determined. The reduction is then possible in $\infty^{k}$ ways.

Continuing this process step by step, we arrive at the general

Theorem. A collineation of type $S_{k+1}$ defined by the formulae

$$
\begin{aligned}
S_{k+1}: & \rho y_{i}=m_{i} x_{i} & (i=1,2, \cdots, k+1), \\
\rho y_{i} & =x_{i} & (i=k+2, \cdots, n+1),
\end{aligned}
$$

where $m_{1} m_{2} \cdots m_{k+1}=(-1)^{k+1}$ can be resolved into a product of $k+1$ perspective reflections $T_{1} T_{2} T_{3} \ldots T_{k+1}$ in $\infty^{k^{2}}$ ways. If these reflections be subject to the conditions

$$
\left(T_{1} T_{2}\right)^{3}=1, \quad\left(T_{1} T_{2} T_{3}\right)^{4}=1, \text { etc. }
$$

the reduction can be effected in $\infty^{\mathrm{j}^{k(k+1)}}$ ways. Moreover, it is clear that $k+1$. is the minimum number of reflections involved in the reduction.

The general collineation in space of $n$ dimensions is included in the theorem; it is only necessary to let $k=n$. 